Cahiers de la recherche sur les droits fondamentaux

Recherche sur les Droits Fondamentaux

$11 \mid 2013$

Le droit de la famille en (r)évolutions

\title{
Réflexions sur la filiation adoptive dans le cadre de la loi ouvrant le mariage aux personnes de même
} sexe

Annick Batteur

\section{OpenEdition}

Journals

Édition électronique

URL : https://journals.openedition.org/crdf/4630

DOI : $10.4000 /$ crdf. 4630

ISSN : 2264-1246

Éditeur

Presses universitaires de Caen

Édition imprimée

Date de publication : 1 novembre 2013

Pagination : $39-44$

ISSN : $1634-8842$

Référence électronique

Annick Batteur, « Réflexions sur la filiation adoptive dans le cadre de la loi ouvrant le mariage aux personnes de même sexe ", Cahiers de la recherche sur les droits fondamentaux [En ligne], 11 | 2013, mis en ligne le 01 décembre 2014, consulté le 15 novembre 2022. URL : http://journals.openedition.org/ crdf/4630 ; DOI : https://doi.org/10.4000/crdf.4630 


\title{
Réflexions sur la filiation adoptive dans le cadre de la loi ouvrant le mariage aux personnes de même sexe
}

\author{
Annick BATTEUR \\ Professeure de droit privé à l'Université de Caen Basse-Normandie \\ Centre de recherches en droit privé (CRDP, EA 967)
}

I. L'adoption simple, modèle d'adoption pour l'enfant rattaché aux couples de personnes de même sexe

A. L'adoption simple et la double parenté réelle ou symbolique

B. L'adoption plénière et l'absence de dimension symbolique de la parenté sexuée

II. L'adoption simple, modèle de filiation pour l'enfant rattaché aux couples de personnes de même sexe

A. L'interdit de l'assistance médicale à la procréation (AMP) en faveur des femmes homosexuelles, un interdit non sanctionné

B. L'interdit de la procréation et de la gestation pour le compte d'autrui, un interdit sanctionné relatif à tous les couples

La loi du 17 mai $2013^{1}$ ouvrant le mariage aux personnes du même sexe, validée par le Conseil constitutionnel ${ }^{2}$, tend à abolir dans le droit du mariage la condition tirée de la différence de sexe et à ouvrir l'adoption aux couples de gays et lesbiennes ${ }^{3}$. À l'interdit absolu qui dominait jusqu'alors, est substitué un droit pour les homosexuels de se marier et d'adopter des enfants. Les critiques se déchaînent. Les homosexuels peuvent désormais se marier, et avoir des enfants par la voie de l'adoption, et c'est ce droit et lui seul qui a été et est encore au cœur des débats actuels les plus virulents. On aurait pu se contenter de reconnaître aux homosexuels le droit d'être des parents, investis de pouvoirs éducatifs. Il suffisait alors d'adapter les textes sur la délégation d'autorité parentale. On aurait raisonné en termes de parentalité et non de parenté ${ }^{4}$. À supposer qu'il fût envisagé, ce choix n'a pas été retenu; les rédacteurs du projet de loi ont été beaucoup plus ambitieux. Ils ont voulu reconnaître le droit aux homosexuel(le)s de devenir parents, et cela grâce à l'adoption, ouverte sans aucune distinction entre adoption simple et adoption plénière. L'Assemblée nationale est allée en ce sens et, en dépit des suggestions faites par des juristes dont nous sommes lors

1. Loi $\mathrm{n}^{\circ} 2013-404,17$ mai 2013, JORF, 18 mai 2013, p. 8253.

2. CC, déc. $\mathrm{n}^{\circ}$ 2013-669 DC du 17 mai 2013.

3. Le Sénat a adopté, dans la soirée du mardi 9 avril, le premier article du projet de loi sur le mariage homosexuel, le plus important, qui ouvre le mariage aux personnes de même sexe. Cet article a été adopté par 179 voix contre 157, sans modification par rapport au vote en première lecture de l'Assemblée nationale. Ce vote devient donc définitif sauf si l'ensemble du projet de loi était rejeté à l'issue de son examen par le Sénat.

4. Voir notamment Mariage-conjugalité, parenté-parentalité, H. Fulchiron (dir.), Paris, Dalloz (Thèmes et commentaires), 2009. 
des auditions devant l'Assemblée nationale ${ }^{5}$ et devant le Sénat ${ }^{6}$, ce sont les deux formes d'adoptions qui sont ouvertes. À plusieurs reprises, il a été dit haut et fort par des juristes, mais pas seulement par eux, que l'adoption devait préalablement faire l'objet d'une réforme législative: cela n'a pas été le cas. Il est même fort probable qu'aucune réforme sérieuse du droit de la filiation ne sera menée dans les mois à venir, en ces temps où la majorité gouvernementale ne peut guère s'offrir le luxe d'un deuxième grand débat de société.

Pouvait-on, avant qu'il ne soit trop tard, imaginer que le législateur dans sa grande sagesse, limite l'accès aux personnes de même sexe à la seule adoption simple? Autrement dit, à supposer que le mariage homosexuel soit institutionnalisé, la loi pouvait-elle se permettre d'exclure les gays et lesbiennes de toute possibilité de recourir à l'adoption plénière d'un enfant? À peine posée, la question suscite des réserves. L'objectif de la réforme est d'assurer une égalité de traitement entre les personnes hétérosexuelles et homosexuelles. $\mathrm{La}$ « discrimination» que l'on supprime au niveau du mariage serait immédiatement réintroduite au niveau de la filiation? Quelle incohérence à première vue! Est-ce même envisageable au regard de la jurisprudence de la Cour européenne des droits de l'homme? Dans un arrêt au fort retentissement ${ }^{7}$, la Cour EDH dans une décision du 15 mars 2012 concernant la France a jugé que l'article 12 de la Convention n'impose pas aux gouvernements des États parties l'obligation d'ouvrir le mariage à un couple homosexuel. Le droit au mariage homosexuel ne peut pas non plus se déduire de l'article 14 combiné avec l'article 8. De plus, lorsque les États décident d'offrir aux couples homosexuels un autre mode de reconnaissance juridique, ils bénéficient d'une certaine marge d'appréciation pour décider de la nature exacte du statut conféré $(\$ 108)$.

Plus récemment, dans l'affaire $X$. et autres c. Autriche du 19 février $2013^{8}$, le cœur du contentieux s'est cristallisé essentiellement autour de la comparaison de la situation du couple homosexuel avec celle d'un couple hétérosexuel non marié. En effet, à la différence du droit français tel que jugé dans l'affaire Gas et Dubois c. France,

[...] en droit autrichien l'adoption coparentale est ouverte non seulement aux couples mariés, mais aussi aux couples hétérosexuels non mariés, alors qu'elle est juridiquement impossible pour les couples homosexuels ${ }^{9}$.

Appréciant si la situation des requérants est comparable à celle d'un couple hétérosexuel non marié dont l'un des membres aurait souhaité adopter l'enfant de l'autre, la Cour répond positivement en soulignant qu'

[...] en termes de personnes, les couples homosexuels et les couples hétérosexuels sont en théorie tout aussi aptes ou inaptes les uns que les autres à l'adoption en général et à l'adoption coparentale en particulier ${ }^{10}$.

La Cour indique que seules « des raisons particulièrement solides et convaincantes ${ }^{11}$ (telles que la préservation de la famille traditionnelle ou la protection des intérêts de l'enfant) peuvent permettre d'exclure des couples homosexuels du champ de l'adoption coparentale ouverte aux couples hétérosexuels non mariés. Certes, il n'est pas impossible d'introduire une différence de traitement, mais il est manifeste que la Cour va de plus en plus exiger les mêmes droits pour les couples mariés quel que soit le sexe des époux.

Dès lors, on ne peut guère concevoir de limiter l'adoption à la seule adoption simple pour les homosexuels, les hétérosexuels, eux, pouvant continuer à bénéficier d'une adoption plénière. Si on tire des enseignements de la jurisprudence de la Cour européenne, on peut estimer que dès qu'il y a mariage, il est difficile de distinguer entre les cas d'adoption pour toutes les personnes qui se trouveront liées par un mariage. Le législateur français ne pouvait donc prendre le risque d'interdire aux homosexuels d'adopter des enfants en la forme plénière.

Pour autant, tout n'est pas réglé, car c'est du côté du pouvoir judiciaire que la problématique va rejaillir. Une adoption plénière ne peut être prononcée que si elle est de l'intérêt de l'enfant. Le juge saisi d'une telle demande peut refuser de la prononcer, et opter pour une adoption simple. Dans quelques mois, les juges saisis de demandes émanant de couples homosexuels ou du conjoint du parent de l'enfant vont devoir prendre parti. Or, à y réfléchir, de nombreux arguments peuvent être avancés pour limiter l'adoption à la forme simple. L'adoption est soumise à un contrôle a posteriori qui permet une vérification concrète de l'intérêt de l'enfant: les juges pourraient avoir

5. E. Binet, Rapport fait au nom de la commission des lois constitutionnelles, de la législation et de l'administration générale de la République sur le projet de loi ( $\left.n^{\circ} 344\right)$, ouvrant le mariage aux couples de personnes de même sexe, t. II, Contributions écrites des personnes entendues par le député rapporteur, $\mathrm{n}^{\circ}$ 628, enregistré à la présidence de l'Assemblée nationale le 17 janvier 2013, p. 597-608.

6. J.-P. Michel, Rapport fait au nom de la commission des lois constitutionnelles, de législation, du suffrage universel, du Règlement et d'administration générale sur le projet de loi, adopté par l'Assemblée nationale, ouvrant le mariage aux couples de personnes de même sexe, t. II, Contributions écrites des personnes entendues par le député rapporteur, no 437, enregistré à la présidence du Sénat le 20 mars 2013 , passim.

7. Cour EDH, Gas et Dubois c. France, 15 mars 2012, req. n 25951/o7. Voir N. Hervieu, «Pusillanimité jurisprudentielle et carences conventionnelles à l'heure d'assurer l'égale protection des cellules familiales homoparentales», Lettre "Actualités Droits-Libertés» du CREDOF, 16 mars 2012.

8. Voir N. Hervieu, «Un long chemin européen vers la pleine reconnaissance des familles homoparentales», Lettre «Actualités Droits-Libertés» $d u$ CREDOF, 26 février 2013.

9. Cour EDH, X. et autres C. Autriche, 19 février 2013, req. nº 19010/07, $\$ 111$.

10. Ibid., $\$ 112$.

11. Ibid., $\$ 88$. 
tendance à juger que le type de lien de filiation électif résultant d'une adoption, bien qu'autorisé largement par la loi, n'est conforme à cet intérêt que s'il n'empêche pas l'établissement d'une filiation biologique d'origine.

Notre système juridique en matière de droit des personnes mais aussi en matière de filiation a un fondement spécifique au regard des sexes: chaque sexe a une place qui lui est réservée. On est homme ou femme (on naît homme ou femme... avec tous les problèmes juridiques suscités par la question de l'identité sexuelle et le genre), mais aussi, en tant que parent, père et mère ont chacun vocation à avoir une place. Le lien de parenté n'est pas nécessairement l'expression d'une vérité absolue quant à la procréation, mais il reflète une insertion dans un système où chacun, homme et femme, a naturellement sa place. Comme l'a écrit un auteur, «Il y aurait là l'expression d'un choix anthropomorphique découlant de ce que les systèmes de parenté appartiennent aux grandes institutions productrices de sens ${ }^{12}$. Dans cette analyse, l'adoption simple peut sembler une bizarrerie, ou si l'on préfère, une exception à un système de référence, puisque l'enfant peut avoir deux pères ou / et deux mères. Depuis que cette forme d'adoption existe, elle a pu être utilisée par des homosexuels au bénéfice d'enfants majeurs. L'établissement d'une parenté à l'égard de deux personnes du même sexe existe déjà.

De tout cela résulte la nécessité d'insister sur cette question de l'adoption des enfants par des homosexuels. Dès lors que l'on conçoit la filiation dépendante de l'altérité des sexes - ce qui était une évidence hier, et ne l'est plus aujourd'hui -, un compromis est envisageable, qui consiste à limiter l'adoption d'un enfant par des couples du même sexe à la seule adoption simple (I). Seul ce choix, qui à défaut d'être politique sera judiciaire, permettra indirectement de respecter autant que faire se peut les interdits quant aux autres modes d'établissements de la filiation, et par là évitera une transformation radicale des fondements traditionnels du droit de la filiation (II).

\section{L'adoption simple, modèle d'adoption pour l'enfant rattaché aux couples de personnes de même sexe}

L'adoption est une institution: elle n'existe que par la loi; c'est un engagement solennel de devenir parent(s), soumis à un contrôle administratif ${ }^{13}$ et juridictionnel. Deux modalités d'adoption existent, qui tendent toutes les deux à l'établissement d'un lien juridique de filiation, mais avec des finalités différentes. Alors que l'adoption simple maintient les liens de l'enfant avec sa famille d'origine, l'adoption plénière rompt ce lien. Admettre l'adoption simple d'un enfant par un couple homosexuel marié (ou par le conjoint homosexuel), c'est introduire l'enfant dans une famille homosexuelle, mais en maintenant les liens biologiques, au minimum sur le plan symbolique, avec la personne de l'autre sexe dont l'enfant est issu. Admettre l'adoption plénière d'un enfant par un couple homosexuel marié, c'est le rattacher à deux branches de même nature, des branches maternelles exclusivement ou des branches paternelles exclusivement, et ainsi le couper pour toujours de ses origines bisexuées et le priver «de la moitié de son ascendance ${ }^{14}$.

\section{A. L'adoption simple et la double parenté réelle ou symbolique}

L'adoption simple se distingue radicalement de l'adoption plénière par ses effets: elle ne rompt pas les liens de l'adopté avec sa famille d'origine. Elle maintient un lien de famille et en crée un nouveau. Si, par exemple, une femme a eu un enfant d'une précédente union, puis, qu'affichant clairement son homosexualité, elle décide de se marier avec une femme, l'adoption simple permet(trait) à sa conjointe du même sexe de devenir officiellement la seconde mère de l'enfant, sans pour autant «gommer» le lien avec le père. L'enfant reste rattaché à ses deux parents biologiques lorsque son père l'a reconnu spontanément. Ainsi, en cas d'adoption simple, l'identité de l'enfant fondée sur une fécondation sexuée apparaît clairement et devrait à l'avenir continuer à apparaître. Son acte de naissance affiche aujourd'hui ostensiblement deux rubriques: «père»/ «mère»- même si l'une des deux reste libre. Le jugement d'adoption est simplement mentionné en marge de l'acte de naissance. L'adoption simple réalise ainsi un équilibre entre les deux familles: famille par le sang et famille adoptive, au moins en principe, car elle donne tout de même la primauté au lien d'adoption pour l'autorité parentale (les deux femmes exerceront ensemble cette autorité).

Si l'enfant n'avait pas officiellement de père avant l'adoption, la situation serait-elle la même en ce sens que le père biologique de l'enfant, voire même un père «social ", pourrait ensuite établir un lien de filiation par voie de reconnaissance ou de possession d'état? L'épouse de la mère de l'enfant sans paternité pourrait-elle, en adoptant

12. P. Murat, «Prolégomènes à une hypothétique restructuration du droit des filiations», in Mélanges en l’honneur du professeur Jean Hauser, P. Delmas Saint-Hilaire, A. Gouttenoire, M. Lamarche et al. (dir.), Paris, LexisNexis - Dalloz, 2012, p. 406. Voir aussi, notamment, C. Neirinck, «Mariage homosexuel: faut-il tenir compte du sexe des êtres humains?», Droit de la famille, décembre 2012, repère $n^{\circ}$ 12, p. 1; F. Millet, «La paternité: chronique d'une mort annoncée», Dalloz, 2013, Point de vue, p. 782.

13. L'agrément est une condition légale circonscrite aux requêtes concernant l'adoption de personnes mineures. Voir Code de l'action sociale et des familles, art. L. 225-2 et L. 225-17.

14. C. Neirinck, «Filiation par convenance et droits de l'enfant», Droit de la famille, avril 2013, repère $\mathrm{n}^{\circ} 4$, p. 1. 
l'enfant de son conjoint, prendre de manière définitive la place du père? Les textes nouveaux ne modifient pas les règles sur l'adoption simple sur ce point: la règle est encore et toujours que l'adoption simple laisse subsister le lien de filiation d'origine. L'enfant aura deux mère(s) et peut-être un père, dans le présent ou le futur. Dans le cas où la paternité n'aurait pas été établie, l'enfant garderait la faculté d'avoir officiellement un père si la paternité, alors vacante, restait vierge.

\section{B. L'adoption plénière et l'absence de dimension symbolique de la parenté sexuée}

L'adoption plénière est beaucoup plus radicale. Elle évince la filiation d'origine, qui est «effacée». Dans le cas d'une adoption par deux homosexuel(le)s, cet effacement est particulièrement grave, puisque la filiation biologique d'origine DISPARAÎTT au bénéfice d'une filiation asexuée. Dans notre exemple, le père biologique n'existe plus; seule compte la nouvelle famille. L'enfant a deux mères; il n'a jamais eu de père. Au niveau des actes de l'état civil, comment va-t-on faire? Aujourd'hui, la filiation adoptive est calquée sur le modèle de la filiation biologique. Tout doit se passer comme si les parents adoptifs étaient les parents biologiques de l'enfant. L'acte de naissance de l'enfant est annulé, et l'on fabrique un acte de naissance pour l'enfant, à partir du jugement d'adoption, avec deux rubriques "père»/ «mère». Que fera-t-on en cas d'adoption par des homosexuel(le)s? Indiquera-t-on «Parent 1 » et «Parent 2 »? Le projet de loi renvoie à un décret la question de l'acte de naissance, ce qui est un tort, car se joue, à travers la détermination de sa filiation sur les actes de l'état civil de l'enfant, la question de ses origines officielles et de son identité. Au cours des auditions devant les deux assemblées, nous avons dénoncé l'absence de toute disposition dans les projets sur ce point. C'est par décret que sera réglé ce problème, ce qui nous paraît discutable. Il est vraisemblable que les actes de l'état civil de l'enfant rattaché par une adoption plénière à un couple d'homosexuel(le)s ne feront aucune allusion à la différenciation sexuelle, et que sur les actes de l'état civil, n'apparaîtra qu'un rattachement exclusif à deux mères, sans allusion à la paternité. On sera dans un cas de pure fiction juridique.

Pourra-t-on imaginer qu'un juge prononce une adoption plénière dans de telles conditions? Peut-il être de l'intérêt de l'enfant d'être rattaché officiellement à deux femmes, ou éventuellement deux hommes, sans que son état civil ne laisse place à un référent paternel ? Bien sûr, dans les faits, l'enfant saura nécessairement qu'il a été conçu d'un homme et d'une femme. Mais est-il de son intérêt d'avoir un acte de naissance qui fait abstraction de cette réalité? Il s'agit non seulement d'effacer toute l'histoire de l'enfant, comme dans une adoption classique par deux hétérosexuels, mais de lui supprimer les racines de ce qui fait son identité, à savoir le fait qu'il est issu de la rencontre de gamètes d'un homme et d'une femme, ce qui est le point commun à toutes les personnes, quelle que soit leur sexualité.

Le modèle même de l'adoption plénière est aujourd'hui très discuté. La difficulté extrême pour les enfants d'avoir accès à leurs origines personnelles (notamment pour les enfants nés sous $\mathrm{X}$ ) conduit déjà à rendre ce type d'adoption très discutable. L'évolution à venir est vers la transformation de ce modèle familial, mais rien ne se fera avant des mois, peut-être même des années. Il aurait fallu réformer le droit de l'adoption, notamment de l'adoption plénière, avant de résoudre la question de son utilisation au bénéfice des couples de même sexe, et préciser les règles en matière de rédaction des actes de l'état civil, en faisant apparaître, quelle que soit la situation, la double origine biologique de l'enfant.

Entre deux formes d'adoption, simple ou plénière, le juge aura à trancher. Nous doutons qu'il soit de l'intérêt de l'enfant qu'une adoption plénière soit prononcée. L'enfant aura pour toujours une seule famille composée de deux femmes (ou deux hommes), famille unilinéaire, et aura en plus un état civil totalement fictif. Pourquoi faudrait-il que sa famille élective exclue à jamais une autre famille? N'est-il pas plus sage de réserver l'avenir, en laissant la possibilité d'établir la filiation biologique, ou au moins de la préserver, dans l'ordre symbolique?

Si tel était le choix de société à laquelle l'application concrète de la loi nouvelle devait conduire, la réforme n'aurait finalement qu'un impact bien limité. L'abandon de la différence de sexe en cas d'adoption ne sera réel que si les juges acceptent de prononcer des adoptions plénières, et tout milite à notre avis pour exclure le prononcé de telles adoptions, cela d'autant plus que les autres modes de filiations n'ont pas été modifiés, et ne le seront pas: le droit de la filiation reste fondé sur l'altérité sexuelle.

\section{L'adoption simple, modèle de filiation pour l'enfant rattaché aux couples de personnes de même sexe}

Il existe aujourd'hui trois modes de filiation et l'on s'interroge sur le devenir de chacune d'elles dans le cadre de la parenté homosexuelle. Quelle sera la construction juridique qui l'emportera? À supposer que l'on ne crée pas un nouveau droit de la filiation (et il faut être réaliste: c'est probablement ce qui se passera), on se doit bien sûr de réfléchir aux conséquences du droit qui est désormais le nôtre sur les modes d'établissement de la filiation autres que l'adoption.

On peine à dénommer ce qui autrefois relevait de l'appellation commode de «filiation légitime ou naturelle». De cette filiation «charnelle», ou filiation «par nature», le projet de loi ne dit rien, et l'on ne peut bien sûr que s'en féliciter. Ce droit concerne exclusivement l'enfant des hétérosexuels. Les père et mère désignés comme tels par la loi sont les parents biologiques. Les forces génétiques qui ont été source de fécondité proviennent d'un homme et 
d'une femme, et la loi organise la manière dont l'enfant sera rattaché à ceux dont il est issu. La filiation est ici inséparable de la procréation. Tout projet qui tendrait à introduire l'équivalent d'une présomption de paternité en cas de mariage de deux hommes ne saurait qu'être repoussé. Les fictions juridiques ont des limites, et précisément, en matière de famille homosexuelle, c'est l'adoption qui doit servir de base à toute construction juridique. La filiation par nature ne concerne pas les couples mariés homosexuels.

Nombreux sont ceux qui estiment qu'ouvrir l'adoption aux gays et lesbiennes revient à reconnaître aux couples de même sexe une possibilité d'avoir un enfant que la nature ne peut pas leur donner. On parle de «droit à...»l'enfant, expression bien discutable (marié ou non, homosexuel ou hétérosexuel, qui pourrait prétendre avoir un droit subjectif à «obtenir» un enfant?). Il est irritant de voir reparaître cette idée, qui fut évoquée en son temps en matière de procréation médicalement assistée par ses opposants, lorsqu'il est question d'homosexualité.

La question s'est compliquée en quelques années de par les pratiques des couples homosexuels qui, malgré l'interdiction de recourir en France à la procréation médicalement assistée et à la technique dite autrefois des «mères porteuses », profitent de la disparité des législations nationales pour utiliser ces méthodes procréatives, notamment au Royaume-Uni, en Grèce ou aux États-Unis.

\section{A. L'interdit de l'assistance médicale à la procréation (AMP) en faveur des femmes homosexuelles, un interdit non sanctionné}

L'élimination de la parenté par le sang, inhérente aujourd'hui à l'adoption plénière, impliquerait nécessairement, selon certains, l'ouverture des procréations médicalement assistées aux couples de même sexe. Si deux femmes peuvent adopter un enfant, comment expliquer qu'on leur refuse l'accès à l'insémination artificielle avec donneur? L'interdiction pour les couples de même sexe de recourir à l'AMP avec don de gamètes d'un tiers donneur relève du droit médical. Cette procréation assistée est actuellement enfermée dans des limites très étroites. Elle est aujourd'hui ouverte en cas d'infertilité d'un couple ou lorsque la fécondation in vitro permet d'éviter que ne soit transmise à l'enfant une maladie grave. L'enfant issu de ces pratiques a une filiation construite sur le modèle de la filiation biologique, alors pourtant qu'il s'agit d'une filiation élective. Il a été choisi en 1994, dans le cadre des lois bioéthiques, de faire fi de la réalité, et de ne pas traiter la question de la filiation de l'enfant sous l'angle d'une filiation conventionnelle ${ }^{15}$. Tout se passe comme si l'enfant était l'enfant biologique du couple. On est enfermé dans un modèle ambigu, qui rend incohérent tout l'ensemble de la parenté juridique.

Ouvrir l'AMP aux lesbiennes, qui ne sont ni stériles ni atteintes d'une maladie grave transmissible à un enfant à naître, c'est remettre en cause les bases de la législation actuelle, qui rattache la filiation par procréation artificielle au droit de la bioéthique et repose sur une fiction. La procréation assistée, si on l'ouvrait aux homosexuels, commencerait dit-on, alors, sa dérive vers «la convenance ${ }^{16}$, ce à quoi la France serait aujourd'hui totalement hostile. À une époque de pénurie de dons de gamètes, la proposition bouleverserait l'équilibre actuellement existant.

On ne peut pas ouvrir l'AMP aux homosexuelles sans revoir le fondement même de la construction juridique qui la sous-tend. Le gouvernement, qui fut à certains moments favorables à autoriser le recours à l'APM aux lesbiennes, a fait marche arrière : il est aujourd'hui annoncé que l'on attendra l'avis du Comité national d'éthique, dont on devine à l'avance que la réponse sera négative: l'ouverture de la PMA aux homosexuelles n'est pas pour demain...

Est-ce si dramatique? La jurisprudence s'est accommodée de la situation. Il arrive aujourd'hui que des femmes homosexuelles affirment haut et fort que l'enfant qu'elles élèvent ensemble est issu d'une PMA faite à l'étranger, ceci à l'occasion d'une demande de délégation d'autorité parentale ou d'adoption de l'enfant de l'une d'elles, demandes la plupart du temps rejetées, au nom de la prohibition de l'établissement de la famille homosexuelle ${ }^{17}$, mais jamais sur le motif que les mères concernées ont détourné la loi française. Nulle référence au principe d'indisponibilité de l'état des personnes pour sanctionner celles qui veulent s'investir dans l'éducation de l'enfant biologique de leur compagne. Autrement dit, s'agissant des femmes homosexuelles qui ont eu recours à une AMP faite à l'étranger, nous ne tirons pas de conséquences de l'interdiction légale. Comment faire autrement de toutes les façons? Comment savoir si l'enfant a été conçu par accouplement, par la rencontre des corps, ou s'il a été conçu grâce à un don de spermes au Royaume-Uni? La voie qui aurait consisté à sanctionner celles qui ouvertement avouent que l'enfant a été conçu par AMP à l'étranger aurait été bien risquée. En refusant de tirer des conséquences du caractère illicite du recours à la procréation avec donneur, la Cour de cassation a été pragmatique, et continuera à l'être.

Alors, que se passera-t-il lorsque les couples de femmes homosexuelles auront officiellement le droit d'adopter un enfant? Rien au regard de l'interdit du recours à la

15. Voir J. Hauser, «Un nouveau-né: l’enfant conventionnel», Dalloz, 1996, Chron., p. 182

16. C. Neirinck, «Réforme de la PMA: la création de la famille par convenance personnelle», Droit de la famille, février 2013 , repère $\mathrm{n}^{\circ} 2$, p. 1.

17. Cass., $\mathrm{1}^{\text {re }}$ civ., 24 février 2006, $\mathrm{n}^{\circ}$ 04-17.090; Dalloz, 2006, Jur., p. 897-900, note D. Vigneau; Droit de la famille, avril 2006, comm. no 88, note P. Murat, p. 19; Revue trimestrielle de droit civil, 2006, p. 297 sq., obs. J. Hauser; H. Fulchiron, «Parenté, parentalité, homoparentalité», Dalloz, 2006, Point de vue, p. 876-877; Les grands arrêts de la jurisprudence civile, t. 1, Introduction, personnes, famille, biens, régimes matrimoniaux,

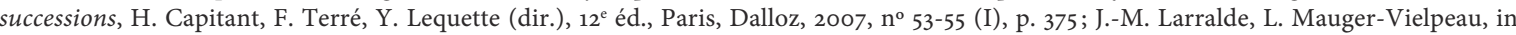
Les grandes décisions du droit des personnes et de la famille, A. Batteur (dir.), Paris, LGDJ-Lextenso, 2012, nº 228, p. 222-232. 
PMA pour les célibataires ou les couples d'homosexuelles. Simplement, désormais, des adoptions simples seront prononcées dès lors que les deux compagnes accepteront de se marier. Solution regrettable, car constituant une incitation à la fraude? Sans doute. Mais tant que l'on ne réformera pas l'ensemble du droit de la filiation, que n'émergera pas un nouveau type de filiation élective, et surtout tant que les dons de spermes seront aussi rares, nous nous adapterons à un système incohérent...

\section{B. L'interdit de la procréation et de la gestation pour le compte d'autrui, un interdit sanctionné relatif à tous les couples}

L'ouverture du droit pour les homosexuel(le)s à fonder une famille par le canal de l'adoption suscite une autre crainte, celle que le droit à la parenté homosexuelle ne remette en cause à moyen terme l'interdit de procréation et de gestation pour le compte d'autrui. Comment être sûr que les couples de même sexe, notamment bien sûr les couples gays, dont la demande d'adoption n'aboutirait pas faute d'enfants adoptables, ne se tournent pas vers la technique dite des "mères porteuses » ? Le risque est grand, dit-on, que des hommes souhaitant avoir un enfant conçu avec leurs propres gamètes ne cherchent à avoir des enfants dans des pays ouverts à ce type de procréation, et demandent ensuite que soit établi ou reconnu le lien de filiation en France, notamment par une adoption plénière. Plus rarement, on évoque le cas des lesbiennes qui, ne pouvant concevoir ou porter un enfant, auraient recours à une gestation ou fécondation rémunérée, à l'étranger. Cette peur est peut-être fondée, encore qu'il faille l'enfermer dans des limites. Il n'y a pas que des homosexuels qui sont tentés d'aller faire à l'étranger ce qui est interdit en France: tous les couples, homos ou hétérosexuels qui ne peuvent avoir d'enfant sont concernés. Cette question de société est grave. Nombreux sont ceux ${ }^{18}$, dont nous sommes, qui pensent qu'il faut absolument maintenir l'interdit posé d'abord par la jurisprudence, puis dans la loi. Les femmes ont beaucoup à perdre à la consécration de ces techniques, qui conduiraient à traiter leur corps comme une marchandise monnayable. Le gouvernement actuel a assuré que cet interdit fondamental ne sera pas remis en cause. Dès lors, les couples gays - et plus rarement les lesbiennes - continueront à être soumis à cet interdit et ne pourront demander l'adoption d'un enfant qu'ils auraient eu par fécondation d'une femme étrangère. Simplement, sur ce point, il est important de rappeler qu'il n'y a pas qu'eux qui sont en cause, et que ce n'est pas parce que l'on ouvre l'adoption aux couples de même sexe qu'il faudra nécessairement lever l'interdit. Les arguments tirés de la nécessaire égalité entre hommes et femmes n'ont ici guère de sens.

Pesant les données en cause, il nous apparaît donc que la filiation par adoption simple est une issue raisonnable aux demandes de ceux ou celles qui veulent offrir à un enfant une place dans leur famille homosexuelle, et ce en l'absence de toute réforme en profondeur du droit de la parenté. Soyons franc: ce n'est pas une solution miracle, et l'on trouvera sûrement à critiquer cette proposition. Elle offre néanmoins un avantage majeur si l'on ferme de plus la voie aux autres modes de filiation: l'enfant de couple de même sexe aura ainsi, comme tout autre enfant, une vocation à la bi-parentalité homme/femme. Que ses parents soient deux hommes, deux femmes, ou un homme et une femme, l'enfant pourra toujours établir sa filiation biologique. Nous ne pensons pas que ceci provoquera un déferlement utilitariste de l'adoption, une submersion qui ravagerait le droit de la filiation. Ou alors, si tel était le cas, ceci obligerait le législateur à revoir les fondements de la parenté, et entraînerait la réforme du droit de la filiation à moyen terme, réforme que tous les spécialistes du droit de la famille appellent de leurs vœux.

18. Voir, parmi une abondante littérature, M. Bandrac, G. Delaisi de Parseval, V. Depadt-Sebag, «Repenser la prohibition de la gestation pour autrui ?», Dalloz, 2008, Chron., p. 434-441; A. Mirkovic, "À propos de la maternité pour autrui», Droit de la famille, juin 2008, étude no 15, p. 9-13 A. Sériaux, «Maternités pour le compte d'autrui: la mainlevée de l'interdit?», Dalloz, 2009, Chron., p. 1215-1220; J. Hauser, «La gestation pour autrui : aspects juridiques et éthiques", in La liberté de la personne sur son corps, P. Muzny (dir.), Paris, Dalloz (Thèmes et commentaires), 2010, p. 85-103. Voir aussi S. Agacinski, Corps en miettes, Paris, Flammarion, 2009, p. 26: «L'usage et la commercialisation des fonctions sexuelles et procréatrices pourraient bien revenir à transformer l'ancienne servitude en travail social moderne». 